\title{
Finite element modelling of inclined screwed timber to timber connections with a large gap between the elements
}

\author{
Coralie Avez $^{1} \cdot$ Thierry Descamps $^{1} \cdot$ Erik Serrano $^{2} \cdot$ Laurent Léoskool $^{1}$
}

Received: 13 February 2015/Published online: 14 January 2016

(C) Springer-Verlag Berlin Heidelberg 2016

\begin{abstract}
Since the 80's, Johanssen's Yield Theory has been adapted to fit new design practices: fastening of timber elements with a layer of insulation (or gap) between the elements or fastening with inclined fasteners. However no rules exist for connections with large gaps (up to 500 $\mathrm{mm})$ and inclined fasteners. The behaviour of screwed connections (timber/large gap/timber) is modelled using an orthotropic material, cohesive surface, Hill criterion and a fictitious material that wraps the screw and models a complex medium where steel and wood interact. The calibrated FE model is finally compared to experimental results.
\end{abstract}

\section{Introduction}

Nowadays self-tapping screws (STS) are largely used in timber construction for fastening and reinforcements (Lathuilliére et al. 2015). STS featuring diameters up to $14 \mathrm{~mm}$ and lengths up to $1000 \mathrm{~mm}$ are produced today (Dietsch and Brandner 2015), what allows for the coupling of high beams and joints on large structures, fastening of CLT walls at the corners or even the fastening of sarking boards. STS mostly feature a continuous thread over the whole length leading to a more uniform load transfer between the screw and the wood material as well as a considerably enhanced axial load-carrying capacity.

Thierry Descamps

thierry.descamps@umons.ac.be

1 Civil Engineering and Structural Mechanics Department, Faculty of Engineering, University of Mons, Mons, Belgium

2 Structural Mechanics Department, LTH, Lund University, Lund, Sweden
Since the 80's, Johanssen's Yield Theory has been adapted to fit new design practices: fastening of timber elements with a layer of insulation (or gap) between the elements or fastening with inclined fasteners (Tomasi et al. 2010). However no rules exist for connections with large gaps (up to $500 \mathrm{~mm}$ ) and inclined fasteners. This paper deals with the FE modelling of such connections. This research is part of a larger project that aims for the design of "sandwich" panels made of two thin parallel CLT panels, fastened with long STS. For the wall conception, the gap between the CLT panels could be filled with an insulated core. For the floor conception, inclined screws are better to transfer shear forces and impede slip between the CLT panels. The gap between CLT panels could be used to improve the dynamic behaviour of the floor or to house cables and technical pipes. Illustration of how such a floor element could look like is presented in Fig. 1.

"Cohesive Surface" is an important evolution in the area of fracture mechanics and finite element modelling. Largely used to study the delamination process in composite, this FE tool has been used with success in different fields of timber engineering (glued-in rods, end-notched beams etc.) (Gabriela et al. 2011; Gonzales et al. 2015). Actually, this cohesive zone does not represent any physical material but describes the cohesive forces which occur when material elements are being pulled apart. The behaviour of screwed connections (timber/large gap/timber) will be fully studied with large experimental campaigns in the future. However, to guide the design of real size floor elements to be tested soon (geometry, diameters and number of screws etc.) the possibility to develop a FE model (ABAQUS) of timber/gap/timber connections, using the "Cohesive Surface" tool has been studied. This paper deals with the definition of this model. 


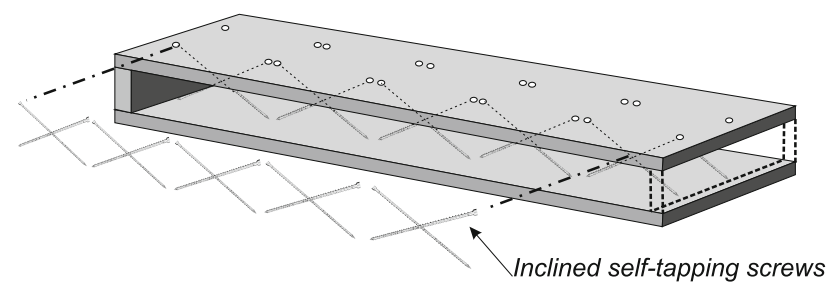

Fig. 1 Sketch of floor element made of two thin CLT panels jointed with inclined STS. Some of the screws are in compression and shear and others are in tension and shear

\section{Materials and methods}

\subsection{Material}

Cross-laminated timber panels are six centimetres thick (3 plies from KLH). Such thin panels are produced by many manufacturers and used by many contractors most of the time for non load-bearing internal walls. Full threaded STS (WR-T-13 from Rothoblaas), $500 \mathrm{~mm}$ long, are used to connect the panels together. The full thread is important to keep the distance between the panels and ensure a good axial strength at both ends of the screws.

\subsection{Methods}

The proposed 3D FE model uses a "Cohesive Surface" to model the interaction between screws and wood, taking into account the brittle behaviour of timber when loaded in shear or in tension perpendicular to the grain. Both parts in contact by means of this surface are "glued" to each other until the shear strength is reached and causes a slip. From the modelling point of view, the proposed model checks the efficiency of this FE tool to model complex interaction between wood and screws (complex loading conditions of the cohesive surface). With the objective of developing a practical model to be used for a first design, some assumptions and simplifications were made:

- To simplify the modelling, the screw is supposed to have a smooth shank of $9 \mathrm{~mm}$ of diameter. This fictitious diameter is defined to get a good compromise between the exact and the modelled tensile rigidity (EA) and flexural rigidity (EI) of the screw $(13 \mathrm{~mm}$ would be too stiff).

- Because of high withdrawal strength of STS, a substratum failure in wood is likely to occur along a cylinder of a diameter of $13 \mathrm{~mm}$ (outer diameter of the threaded part of the screw) or slightly bigger (no steel failure). So, a fictitious material (called "soft material" thereafter) is used to fill the gap between the smooth shank of the modelled screw and the outer diameter of the STS (see Fig. 2). The 3D cylindrical "soft material" that wraps the screw is assumed to be perfectly elastic and has the same mechanical properties as wood. The

FE model with cohesive surface and " soft material »

Fig. 2 Calibration process
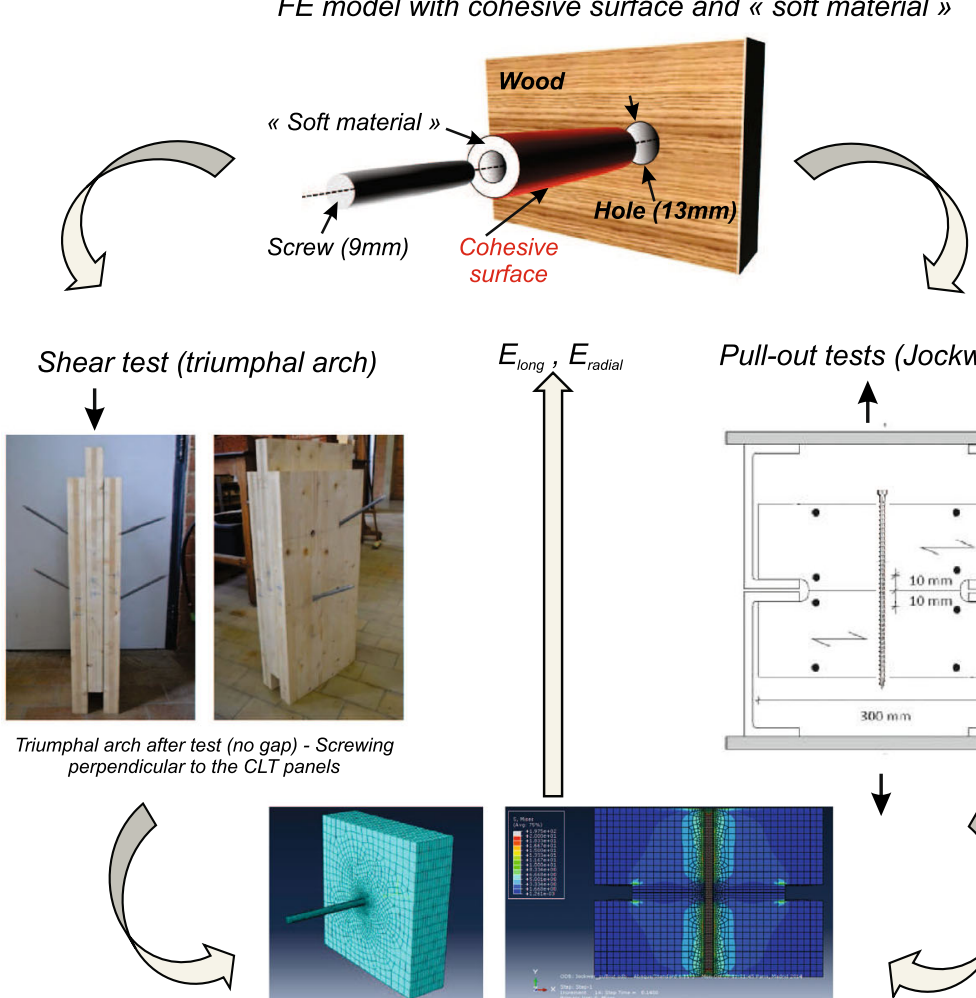
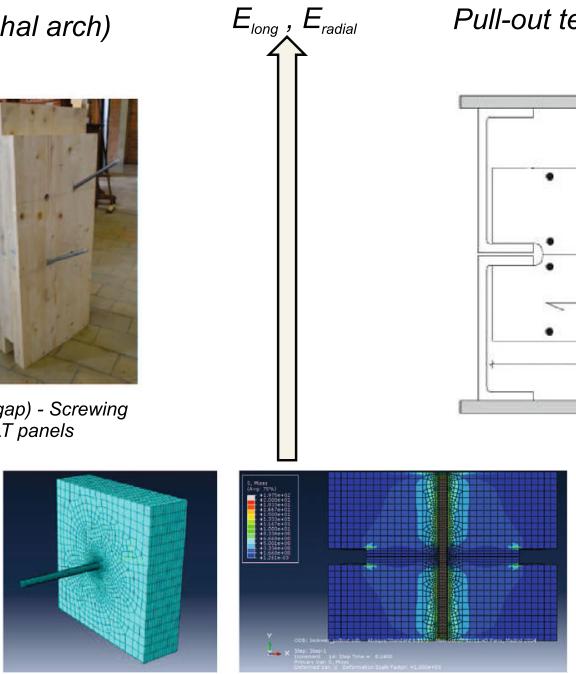

Pull-out tests (Jockwer)
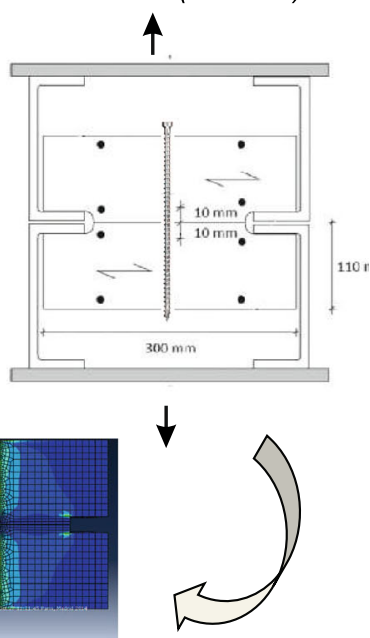

Model calibration 
Table 1 Mechanical properties of the model with a cohesive surface Hill criterion (mean values Statens Byggeforskningsinstitut 1994)

\begin{tabular}{ll}
\hline Longitudinal strength $f_{c / t, 0, \text { mean }}$ & $45 \mathrm{MPa}$ \\
Transverse strength $f_{c / t, 90, \text { mean }}$ & $3 \mathrm{MPa}$ \\
Shear strength $f_{v, 0, \text { mean }}$ & $7 \mathrm{MPa}$ \\
Rolling shear strength $\tau$ & $3.5 \mathrm{MPa}$ \\
Soft material & $50 \mathrm{MPa}$ \\
Radial modulus (MOE) & $370 \mathrm{MPa}$ \\
Other moduli (MOE) & $690 \mathrm{MPa}$ \\
Shear modulus & 0 \\
Poisson coefficient & \\
Cohesive surface & $40 \mathrm{~N} / \mathrm{mm}^{3}$ \\
Longitudinal rigidity & $0 \mathrm{~N} / \mathrm{mm}^{3}$ \\
Tangential and radial rigidities & $5 \mathrm{MPa}$ \\
Shear stress limit & Linear $(0 \mathrm{~N}$ after $4 \mathrm{~mm})$ \\
Damage evolution &
\end{tabular}

elastic properties in the radial and longitudinal directions have been calibrated thanks to lab tests (longitudinal $=$ cylinder axis). These features are fictitious and only used to model a complex medium where steel (part of the thread of the screw) and wood material (around the thread of the screw) interact.

Wood is modelled as an orthotropic material and all the crossed layers of CLT are considered. To model the post yielding behaviour of the connection, steel is modelled as an elastic and perfectly plastic material and the Hill criterion is used for wood (Descamps et al. 2009). The main properties of the model are shown in Table 1. The Hill criterion is dependent on six material parameters and assumes the same yield stress in tension and compression (mean values for spruce are used). The compression and tensile strength are equal and chosen to be the smallest value between both of them. The mean values are used as they allow a better correlation with the experimental tests (characteristic values, especially for tension, are much lower and lead to a premature yielding).

The calibration process of the "soft material" is of course of major importance (Fig. 2). For that purpose, two tests were made to define the elastic properties in the radial and longitudinal directions. On the one hand, one may check that the stiffness of a pull-out test is governed by the "longitudinal" modulus of elasticity (direction of the shank of the screw) of the soft material. The associated failure mode is brittle (cohesive surface is therefore needed to model this behaviour). On the other hand, the stiffness of the connection when loaded in shear is mainly governed by the "radial" modulus of elasticity of the soft material (and the "longitudinal" modulus of elasticity is of minor importance). For this loading configuration, the global behaviour is mainly governed by the bending of the screws and the embedment of the screw into the wood. The failure is ductile and the Hill criterion seems to be sufficient to model the large deformations observed even if the local stress distribution into wood is not relevant. Those both simple configuration tests were used to calibrate the FE model (orthotropic, cohesive surface, "soft material", Hill criterion) as presented in Fig. 2. Properties used for modelling are summarized in Table 1.

\section{Results}

Using a fictitious material is of course a convenient way of doing, however the robustness of the model and its ability to be used in slightly different conditions has to be checked (different diameters, different screwing angles etc.). First of all, the influence of the screwing angle was studied with FE models. A comparison with experimental results
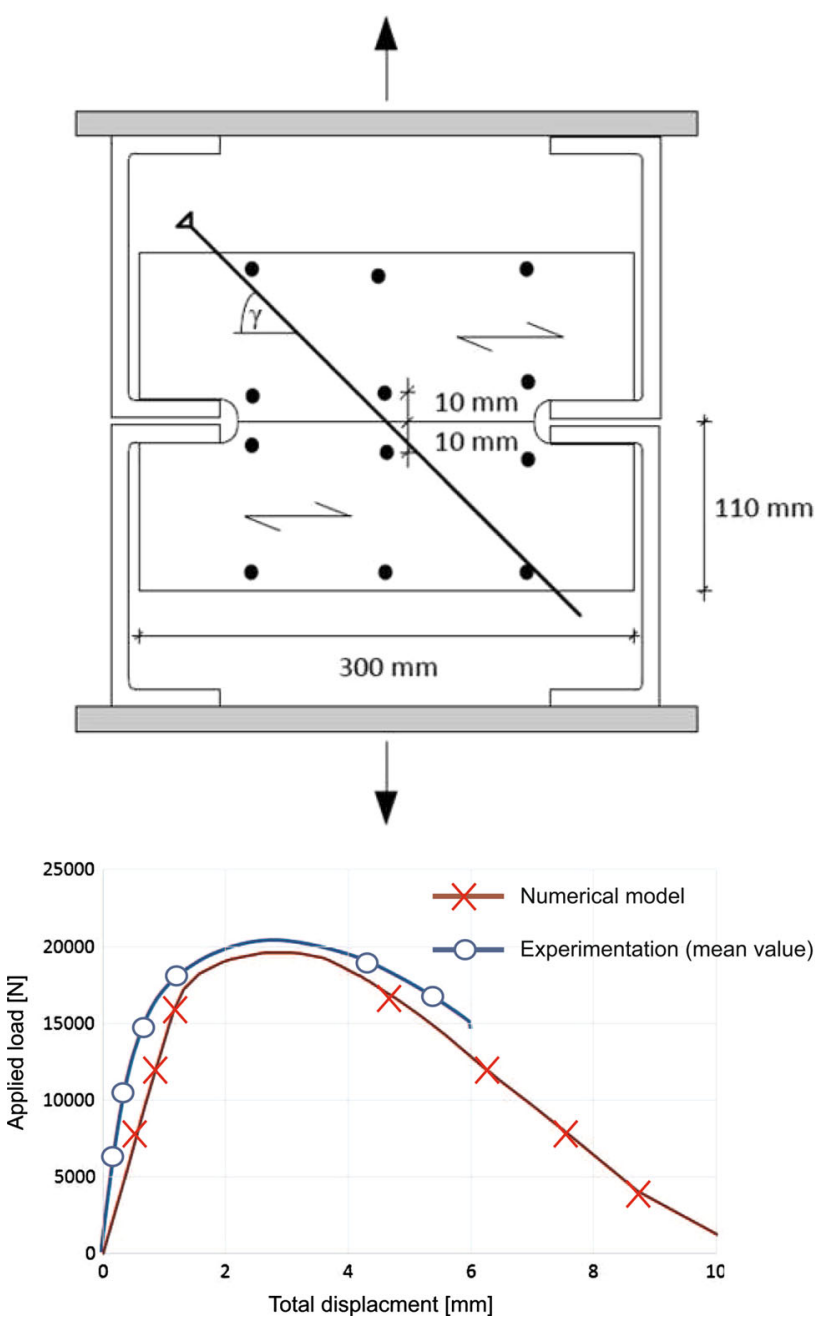

Fig. 3 Example of numerical vs experimental curves (Jockwer experimental tests, Jockwer et al. 2014; Jockwer 2014) 

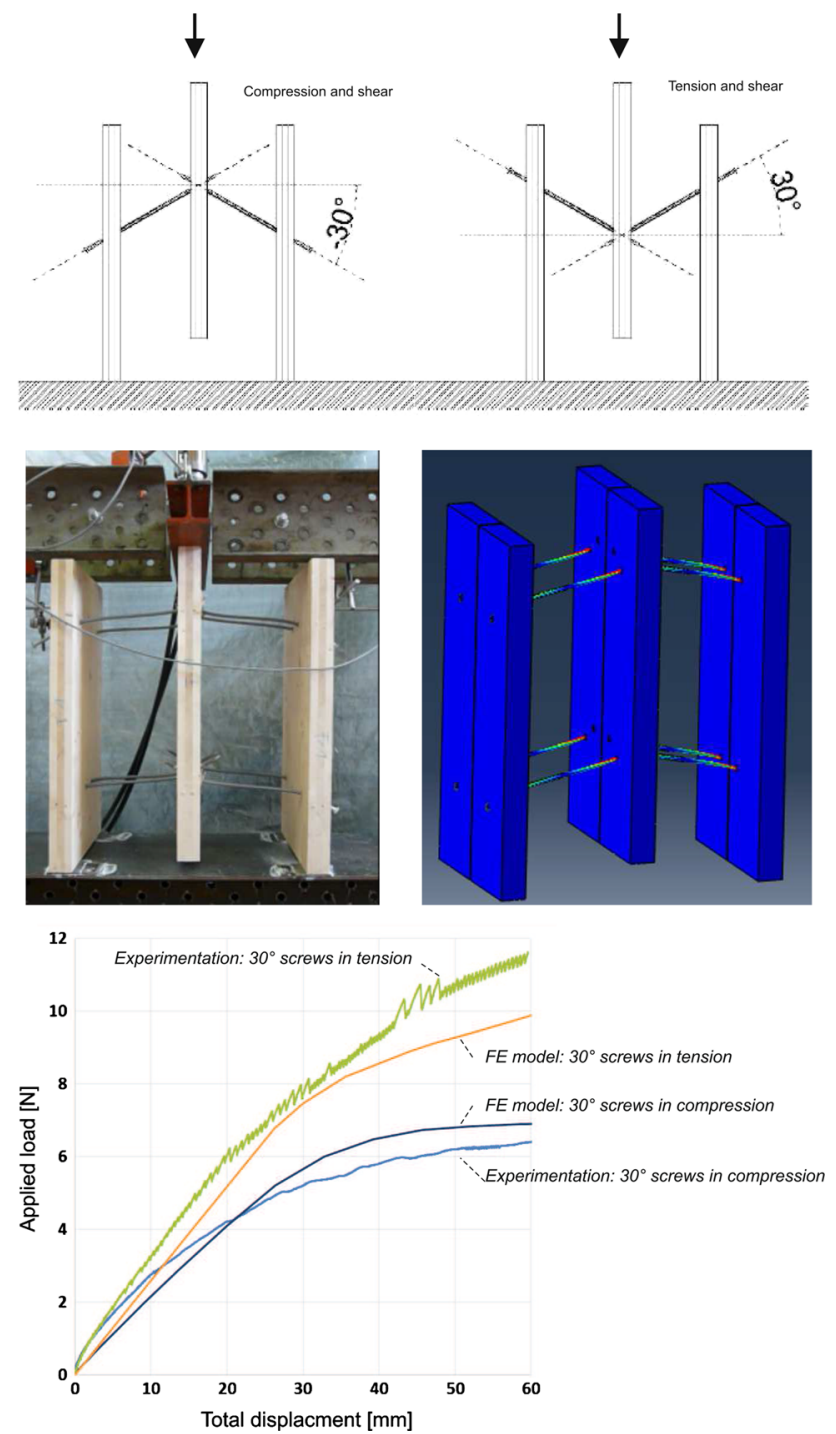

Fig. 4 Triumphal arches with screwing angle of $30^{\circ}$. Experimentations, FEM and comparison of numerical vs experimental curves

coming from the literature is proposed (Jockwer et al. 2014). Figure 3 presents the FE model inspired by Jockwer's tests. The experimental device has been modelled carefully and the properties discussed before have been used. The numerical curve fits well the experimental one whether for the stiffness, the ultimate load or the postfailure behaviour.

Then, screwed timber to timber connections with a large gap were studied by means of specimen fashioned with a triumphal arches shape. Figure 4 shows the experimental device and the FE model of triumphal arches with eight screws (compression and shear) screwed at an angle of $30^{\circ}$. Experimentations highlighted two failure modes for screws when loaded in compression and shear. The first one is the buckling of the screw and the second one is a ductile failure with plastic hinges developing at the interface between timber and insulation. So, that is the reason why a secondorder model was used to allow the buckling of the screws. Figure 4 also presents the comparison between numerical and experimental load displacement curves. Considering the variability of the experimental results and the fact that only few tests were made for this configuration, the correlation seems highly promising. More experimental tests are planned in the near future to validate and calibrate more precisely the numerical models.

\section{Conclusion}

A new method to model a timber to timber connection with a large gap between the joint elements has been developed. Based on the definition and calibration of a fictitious material and the use of a cohesive surface coupled to a common non-linear model, this technique seems to be efficient, robust and quite easy to implement. The "soft material" models a complex area of the connection where wood and steel interact and failure occurs. The methodology proposed for the modelling has allowed to get one set of parameters which fits well all experimental results (triumphal arches, different angles, pull-out etc.). Given that the variability of the experimental tests is not known yet (only a few number of experimental tests have been done), it is difficult to calibrate more accurately the results. Nevertheless, the results are highly promising, with only a slight variation between experimental and numerical results (about $10 \%$ on ultimate limit loads). Further research should improve the model and other screwing configuration must be tested (screwing at $45^{\circ}$, smaller or bigger diameter, etc.). However, one may say that the proposed model already meets basic requirements for the modelling of more complex elements, as for example a floor element made of two parallel thin CLT panels jointed by long self-tapping screws, which is a major step for ongoing research.

Acknowledgments The authors would like to thank COST FP1404 for the support of Mr. Laurent Loskool's stay at Lund University, Sweden, through a "Short Term Scientific Mission" grant, which made this research possible. We would like to extend the warmest thanks to iTechwood, KHL and Rothoblaas companies for their support.

\section{References}

Descamps T, Lalisse D, Datoussaïd S (2009) 3D FEM modeling of slender laterally loaded timber fasteners. In: Curran Associates (ed) Proceedings of the 12th international conference on fracture, July 12-17, Ottawa, Canada

Dietsch P, Brandner R (2015) Self-tapping screws and threaded rods as reinforcement for structural timber elements-A state-of-the- 
art report. Constr Build Mater 97:78-89. doi:10.1016/j. conbuildmat.2015.04.028 (special issue: Reinforcement of Timber Structures)

Gabriela T, Erik S, René S (2011) State-of-the-art review on timber connections with glued-in steel rods. Mater Struct 44(5):997-1020

Gonzales E, Tannert T, Avez C (2015) Timber joints with multiple glued-in steel rods. In: Proceedings of the third international conference on adhesive bonding, Porto

Jockwer R (2014) Structural behaviour of glued laminated timber beams with unreinforced and reninforced notches. $\mathrm{PhD}$ thesis, ETH Zurich

Jockwer R, Steiger R, Frangi A (2014) Fully threaded self-tapping screws subjected to combined axial and lateral loading with different load to grain angles. In: Aicher S, Reinhardt HW,
Garrecht H (eds) Materials and joints in timber structures, vol 9. Springer, The Netherlands, pp 265-272

Lathuilliére D, Bléron L, Descamps T, Bocquet JF (2015) Reinforcement of dowel type connections. Constr Build Mater 97:48-54. doi:10.1016/j.conbuildmat.2015.05.088 (special issue: Reinforcement of Timber Structures)

Statens Byggeforskningsinstitut - Danish Building Research (1994) Trækonstruktioner: Beregning. SBIanvisning - Danish Standard (in Danish)

Tomasi R, Crosatti A, Piazza M (2010) Theoretical and experimental analysis of timber-to-timber joints connected with inclined screws. Constr Build Mater 24(9):1560-1571 